\title{
Using neuroscience for naming psychotropic drugs
}

\author{
Stephen M. Stahl
}

Currently, psychotropic drug "vocabulary" is based on clinical indications, mostly 6 classes, namely, antipsychotic, antidepressant, mood stabilizer, stimulant, anxiolytic, and hypnotic. ${ }^{1,2}$ This naming system creates much confusion, since so-called antidepressants are commonly used as anxiolytics, antipsychotics increasingly as antidepressants, and so on. ${ }^{1,2}$ Vocabulary based on clinical indications also leads to great difficulty in classifying new agents, especially ones with novel mechanisms of action or novel clinical uses. Thus, there has long been a need to rationalize the naming of psychotropic drugs, and a recent task force of experts from psychopharmacology societies around the world (Table 1) is showing the way forward, with Neuroscience based Nomenclature $(\mathrm{NbN}){ }^{3-5}$

So, what is NbN? First and foremost, $\mathrm{NbN}$ names the more than 100 known psychotropic drugs by one of 11 pharmacological domains that include well known terms such as serotonin, dopamine, acetylcholine, and GABA. Included also in $\mathrm{NbN}$ are 10 familiar modes of action, such as agonist, antagonist, reuptake inhibitor, and enzyme inhibitor. $^{3-5} \mathrm{NbN}$ also has 4 additional dimensions or layers. ${ }^{3-5}$ The first layer enumerates the official indications as recognized by the regulatory agencies (ie, the U.S. Food and Drug Administration, the European Medicines Agency, and other governmental organizations). The second layer states efficacy based on randomized controlled trials or substantial, evidence-based clinical data, as well as side effects-not the exhaustive list provided in product monographs, but only the most common ones. The third layer is composed of practical notes. The committee used this section to highlight important drug interactions, metabolic issues, and specific warnings. Finally, the neurobiological effects in laboratory animals and humans are summarized in the fourth section. Specific doses and titration regimens are not provided, as they can vary in different countries.

TABLE 1. Organizations on the task force for Neuroscience based Nomenclature ( $\mathrm{NbN})$

ACNP (American College of Neuropsychopharmacology) AsCNP (Asian College of Neuropsychopharmacology) CINP (International College of Neuropsychopharmacology)

ECNP (European College of Neuropsychopharmacology)

IUPHAR (International Union of Basic and Clinical Pharmacology)
How does $\mathrm{NbN}$ work in practice? The major journals in the field have already begun adapting $\mathrm{NbN}$ for their published papers, and CNS Spectrums is joining them. Specifically, journals adapting $\mathrm{NbN}$ will require authors to clarify the meaning of the terms they use for drugs by defining the term such as "antipsychotic" along with the $\mathrm{NbN}$ term (eg, dopamine D2 antagonist) when the drug name first appears in the main text of the article. Furthermore, to make all new papers searchable by $\mathrm{NbN}$ terminology, the $\mathrm{NbN}$ nomenclature of the various drugs that the article covers should be added to the keywords of the article. The new keywords thus will include those relevant 11 pharmacological domains and 10 modes of action of the drugs discussed in the article. This will make the process easier for authors and readers searching for drugs in our publications. To "translate" between old and new nomenclature, the easiest and most recommended way is to use the approved app, which is available on the project's Web site (http://nbnomenclature. org/), as well as in the software repositories of the various platforms for which it is available. In this Web site, there is a special tab: For Authors. A link to these resources will now be included in CNS Spectrums? Instructions for Authors. The recommended way to use the $\mathrm{NbN}$ is via a specific free app that can be downloaded from Google play (https://play.google.com/store/ apps/details?id=il.co.inmanage.nbnomenclature), iTunes (https://itunes.apple.com/us/app/nbn-neurosciencebased-nomenclature/id927272449?mt=8), or the $\mathrm{NbN}$ website http://nbnomenclature.org/.

What is the current status of NbN? Apart from the 5 international organizations endorsing $\mathrm{NbN}$, and the chief editors of 22 scientific journals supporting the development of this classification system for eventual implementation within the scientific literature, a variety of symposia at international meetings, including the annual meeting of the American Psychiatric Association, are planned for 2016 to present the $\mathrm{NbN}$ to scientists and clinicians.

\section{Disclosures}

Members of the task force (including the author of this editorial) and their organizations have not received financial compensation for their contribution. Costs for 
publication of the book and development/update of the app have been paid for by the European College of Neuropsychopharmacology.

\section{REFERENCES:}

1. Nutt DJ. Beyond psychoanaleptics-can we improve improve antidepressant drug nomenclature? J Psychopharmacol. 2009; 23(4): 343-345.
2. Stahl SM. Classifying psychotropic drugs by mode of action and not by target disorder. CNS Spectr. 2013; 18(3): 113-117.

3. Zohar J, Stahl S, Moller HJ, et al. A review of the current nomenclature for psychotropic agents and an introduction to the Neuroscience-based Nomenclature. Eur Neuropsychopharmacol. 2015; 25(12): 2318-2325.

4. Zohar J, Stahl S, Moller HJ, et al. Neuroscience Based Nomenclature. Cambridge, UK: Cambridge University Press; 2014.

5. Neuroscience based Nomenclature. Utrecht, the Netherlands: ECNP; 2015. http://nbnomenclature.org/. 